\title{
Idiopathic Intracranial Hypertension Related to Chronic Use of Mesalamine: A Case Report and Literature Review
}

\author{
Dev Mehta ${ }^{\mathrm{a}, \mathrm{c}}$, Mohammed El-Hunjul ${ }^{\mathrm{a}}$, Hussam Yacoub ${ }^{\mathrm{a}, \mathrm{b}}$
}

\begin{abstract}
Idiopathic intracranial hypertension (IIH) is commonly seen related to iatrogenic causes such as excess retinol, tetracyclines, and amiodarone. We report a case of IIH likely related to mesalamine use. A 63-year-old woman presented with transient episodes of diplopia and headaches. She was diagnosed with irritable bowel syndrome and started on mesalamine 8 months prior. Her neurologic examination was significant for papilledema and bilateral partial abducens nerve palsies. Her opening pressure was $27 \mathrm{~cm} \mathrm{H}_{2} \mathrm{O}$. Discontinuation of mesalamine in conjunction with acetazolamide correlated with improvement of her papilledema and diplopia. At 6-month follow-up, she still remained asymptomatic. IIH is likely a rare side effect of mesalamine use likely related to 5-aminosalycilate use. This class of medication typically causes headache so this is likely a more complex version. The mechanism of how this occurs is largely unknown. Patients presenting with new onset headache or diplopia acutely or chronically on mesalamine should have ophthalmologic evaluation urgently to evaluate for IIH.
\end{abstract}

Keywords: Intracranial hypertension; Mesalamine; Papilledema; Pseudotumor cerebri

\section{Introduction}

Idiopathic intracranial hypertension (IIH) is a well characterized entity. It is associated with women of child bearing age who are typically obese. The symptoms associated with IIH are typically holocranial headaches getting worse when laying flat and visual symptoms. The cause is unknown but it is thought to be related to increased cerebrospinal fluid production or impaired resorption. Iatrogenic causes of IIH are seen with medications such as retinol (vitamin A) and its derivatives, amiodarone and tetracyclines. Venous sinus thrombosis can acutely cause intracranial hypertension as well. We present

\footnotetext{
Manuscript submitted June 1, 2019, accepted June 14, 2019

aLehigh Valley Health Network, Allentown, PA, USA

bMorsani School of Medicine, University of South Florida, Tampa, FL, USA ${ }^{\mathrm{c} C}$ Corresponding Author: Dev Mehta, Lehigh Valley Health Network, $1250 \mathrm{~S}$ Cedar Crest Blvd, Suite 405, Allentown, PA 18103, USA.

Email: dgmehta89@gmail.com
}

doi: https://doi.org/10.14740/jnr537 a case of IIH likely secondary to chronic mesalamine use.

\section{Case Report}

A 63-year-old woman was diagnosed with irritable bowel syndrome and started on mesalamine. Eighteen months after the diagnosis, she reported transient episodes of diplopia that gradually worsened in frequency and severity over time. She was also experiencing frequent holocranial headaches upon awaking. She was seen by an optometrist for a routine examination and was referred to an ophthalmologist who confirmed the finding of papilledema. She was therefore sent to our Emergency Department for further evaluation.

On physical examination, the patient was found to have a body mass index (BMI) of 37. As per patient report her weight had not fluctuated much over the past 20 years. Neurologic evaluation revealed bilateral partial abducens nerve palsy. Visual acuity was 20/30 bilaterally. Magnetic resonance imaging of the brain and venography of the head were unremarkable. A lumbar puncture was performed with an opening pressure of 27 $\mathrm{cm}_{2} \mathrm{O}$. Immediately after the procedure, the patient reported significant improvement in both the diplopia and headache. The patient was placed on acetazolamide for suspected IIH and was discharged home. Literature review revealed sparse case reports of IIH related to the use of mesalamine so this agent was discontinued. Three months later, the papilledema completely resolved and acetazolamide was discontinued. Six months after discontinuation of acetazolamide, the patient reported no weight loss and no recurrence of headache or other neurologic symptoms.

\section{Discussion}

IIH has the tendency to occur spontaneously, particularly in obese women between 20 and 50 years of age. The patient presented in this case report is considered obese based on the BMI but was not in the typical age range for IIH. Additionally, she had no significant weight changes for quite some time. In our collective experience, we have not encountered a case of IIH that presented in the sixth decade of life. This prompted us to investigate other etiologies. Several agents have been linked to IIH including tetracycline, amiodarone, steroids, and excess retinol. One of mesalamine's most frequent adverse reactions is headache [1]. This is likely related to one of its components 5-aminosalicylate (5-asa). In our literature search on PubMed and Google Scholar, our case of IIH secondary to the use of 
Table 1. Summary of Cases in Literature That Reports Idiopathic Intracranial Hypertension Related to 5-Aminosalicylate

\begin{tabular}{lllll}
\hline Cases & Age, sex & $\begin{array}{l}\text { Presence of } \\
\text { papilledema }\end{array}$ & Associated neurologic signs and symptoms & Clinical outcome \\
\hline Rosa et al [3] & 23, female & Yes & Headache, blurry vision & $\begin{array}{l}\text { Visual acuity did not return to baseline } \\
\text { despite discontinuation }\end{array}$ \\
$\begin{array}{l}\text { Rottembourg } \\
\text { et al [4] }\end{array}$ & 11, female & Yes & Headache & $\begin{array}{l}\text { Returned to baseline after } \\
\text { discontinuation }\end{array}$ \\
$\begin{array}{l}\text { Sevgi et al [5] } \\
\text { 25, female }\end{array}$ & Yes & $\begin{array}{l}\text { Headache, blurry vision, bilateral abducens nerve } \\
\text { palsies, lower facial nerve palsy on the left }\end{array}$ & $\begin{array}{l}\text { Returned to baseline after } \\
\text { discontinuation }\end{array}$ \\
\hline
\end{tabular}

mesalamine would only be the third one reported in adults [2, 3] with a fourth case reported in the pediatric literature [4]. Rosa et al demonstrated that re-challenging mesalamine due to severe ulcerative colitis caused headache and papilledema again [3]. Sevgi et al did not re-challenge their patient with sulphasalazine due to stability on azathioprine [5]. Rottembourg et al's case demonstrated a temporal relation of symptoms of IIH related to starting and discontinuation of mesalamine [4]. Please refer to Table 1 for a description of the course of the syndrome for each case [3-5].

The exact mechanism of why 5-asa has a relationship with IIH is not clear. However, it is known that headache is the most common and quite a significant side effect of 5-asa and its derivatives. Discontinuation of the offending agent is met with a decrease in headache frequency [6].

Despite the discontinuation of acetazolamide and lack of weight loss at follow-up, our patient's symptoms did not recur, further supporting the notion that there is a relationship between IIH and 5-asa derivatives. We suggest that patients on chronic mesalamine therapy should undergo routine ophthalmologic evaluation for papilledema, particularly those with headaches and/or visual disturbance, and be assessed for IIH in intractable cases.

\section{Conclusions}

Patients presenting with new onset headache or diplopia acutely or chronically on mesalamine should have ophthalmologic evaluation urgently to evaluate for IIH.

\section{Acknowledgments}

None.

\section{Financial Disclosure}

None.

\section{Conflict of Interest}

The authors have no conflict of interest.

\section{Informed Consent}

Informed consent was obtained from the patient.

\section{Author Contributions}

The authors all equally contributed to, had access to and interpreted the data, drafted the manuscript, and approved the final version for submission.

\section{References}

1. Radhakrishnan K, Ahlskog JE, Cross SA, Kurland LT, O'Fallon WM. Idiopathic intracranial hypertension (pseudotumor cerebri). Descriptive epidemiology in Rochester, Minn, 1976 to 1990. Arch Neurol. 1993;50(1):78-80.

2. Schroeder KW. Is mesalamine safe? Gastroenterol Hepatol (N Y). 2007;3(11):878-879.

3. Rosa N, Giamundo A, Jura A, Iaccarino G, Romano A. Mesalazine-associated benign intracranial hypertension in a patient with ulcerative colitis. Am J Ophthalmol. 2003;136(1):212-213.

4. Rottembourg D, Labarthe F, Arsene S, Jonville-Bera AP, Maurage C, Rolland JC. Headache during mesalamine therapy: a case report of mesalamine-induced pseudotumor cerebri. J Pediatr Gastroenterol Nutr. 2001;33(3):337338.

5. Sevgi E, Yalcin G, Kansu T, Varli K. Drug induced intracranial hypertension associated with sulphasalazine treatment. Headache. 2008;48(2):296-298.

6. Alloway JA, Mitchell SR. Sulfasalazine neurotoxicity: a report of aseptic meningitis and a review of the literature. J Rheumatol. 1993;20(2):409-411. 